MARKO SUBOTIĆ, Ph.D. ${ }^{1}$

(Corresponding author)

E-mail: msubota@gmail.com

VLADAN TUBIĆ, Ph.D. ${ }^{2}$

E-mail: vladan@sf.bg.ac.rs

${ }^{1}$ University of East Sarajevo, Faculty of Transport

and Traffic Engineering, V. Mišića 52,

74000 Doboj, Bosnia and Herzegovina

2 University of Belgrade, Faculty of Transport and Traffic

Engineering, V. Stepe 305, 11000 Belgrade, Serbia
Traffic Engineering

Preliminary Communication

Submitted: 5 Aug. 2016

Accepted: 21 June 2017

\title{
CAR EQUIVALENTS DEPENDENCE ON THE LONGITUDINAL ROAD GRADIENT ON TWO-LANE ROADS IN BOSNIA AND HERZEGOVINA
}

\begin{abstract}
The main objective of this paper, based upon the extensive empirical research of free flow in local conditions, is to quantify the unfavourable impact of the flow structure on the road capacity using PCE (Passenger Car Equivalent) values as a function of longitudinal grade. Based on literature reviews and empirical research, it has been proved that the PCE value for all vehicle classes is directly correlated with the road gradient. The PCE values in free flow conditions have been determined for the approved vehicle classes. Based on the measured values, models for determining the average PCE value depending on the upward grade on two-lane roads have been developed. Comparison of the developed models in conditions of free traffic flow with the Highway Capacity Manual (HCM) models has shown lower PCE values in this research. Models for the percentage of PCE values $P C E_{15 \%}, P C E_{50 \%}$ and $P C E_{85 \%}$ have also been established.
\end{abstract}

\section{KEY WORDS}

passenger car equivalents; level of service; two-lane road capacity analysis; traffic model; regression analysis;

\section{INTRODUCTION}

Heavy vehicles hypothetically include all motor vehicles larger than passenger cars and therefore the percentage of heavy vehicles differs on roads and depends on the distance of urban areas, availability of alternative routes and the like. Therefore, the percentage of heavy vehicles is higher on the roads more distant from the urban areas as well as on alternative routes. Heavy vehicles significantly affect vehicle flow by their dimensions and operative characteristics (acceleration, deceleration, manoeuvring, etc.). Dimensions of heavy vehicles occupy a larger space than a passenger car. Heavy vehicles and passenger cars have also significantly different operating characteristics. There are three main factors of vehicle movement that have significant impact on the capacity of sections: vehicle length, characteristics of a vehicle and driver behaviour. As the vehicle length increases, the vehicle will occupy larger road space which will lower the maximum traffic density value. This will have direct negative impact on the resulting road capacity.

Road conditions can be improved by modifications of the rolling coefficient, running friction and vehicle dynamic characteristics, especially for heavy vehicles. Based on Rahka [1] where distinction between seven different road types and pavement conditions was made (concrete pavement excellent condition, concrete pavement good condition, concrete pavement bad condition, asphalt pavement good condition, asphalt pavement proper condition, asphalt pavement bad condition and asphalt pavement under snow), the PCE value on good pavements is less variable. It can be intuitively concluded that as vehicle length increases so does the passing time through the cross section. Research by Kockelman [2] points out the fact that vehicle length has negative influence on the traffic flow. The paper analysed different third order polynomial models which describe relations between traffic flow and density which affects other variables. Also, it has been estimated that the influence of a larger vehicle with respect to average vehicle length is about $17.4 \%$. The average length of combined vehicles and pick-ups is about $14 \%$ bigger than passenger car length, while the average sport vehicle has almost the same length as an average car. When it comes to technical and exploitation vehicle characteristics, the research on acceleration which has a lot of connections with power and mass ratio is always topical. Based on the findings of several studies [3] HCM states that the average ratio of power and mass is between 76.1 and $90.4 \mathrm{~kg} /$ kW (125 and $150 \mathrm{lb} / \mathrm{hp})$.

In many cases during the measurements, it was observed that heavy vehicles which were located in 
front of the passenger cars lead to more cautious and slower driving, because the dimensions of the vehicle itself reduce visibility. This fact has direct impact on the reduction of speed of passenger vehicles. Other factors that have negative influence on the traffic flow include weather conditions, visibility, speed limits, etc. [4]. There is not much research on impact of poor visibility on the traffic flow and therefore appropriate PCE values in such conditions are still not precisely determined. HCM-2000 [3] confirms that bad weather conditions can significantly reduce capacity and exploitable speed and deals with issues like where and how to take these effects into consideration. These are all very important factors which disturb the PCE values.

Based upon the extensive empirical research of free flow in local conditions, the main goal of this paper is to quantify the unfavourable impact of the flow structure on the road capacity using the PCE (Passenger Car Equivalent) values as a function of Iongitudinal grade. The PCE values in free flow conditions have been determined for the approved vehicle classes. Based on the measured values, models for determining average PCE value depending on the upward grade on the two-lane roads have been developed. Comparison of the developed models in conditions of free traffic flow with the Highway Capacity Manual (HCM) models has shown lower PCE values in this research. Models for the percentage of PCE values $\mathrm{PCE}_{15 \%}, \mathrm{PCE}_{50 \%}$ and $\mathrm{PCE}_{85 \%}$ have also been established.

The remaining of the paper is structured as follows. Section 2 gives a brief description of the related work. Methodology for determining PCE at cross section is described in the third Section. Numerical results are given and discussed in Section 4. Finally, in Section 5 we conclude the article, giving managerial implications and directions for further research.

\section{RELATED WORK}

There are different chronological approaches of determining the PCE with respect to different vehicle categories under different road and traffic conditions [5]. Elefteriadou [6] used the average speed as a performance measure while Bhuvanesh [7] used concentration as a traffic characteristic, which is defined as proportion of road space occupied by vehicles at an instant for validating the simulation model. In this study the video graphic technique was used to collect concentration data at 15-second intervals. However, Khan and Maini [8] did an extensive review of heterogeneous traffic flow modelling studies and concluded that for the mixed type of vehicles, linear density measurements are inadequate, and that there should be a measure which includes the vehicle area. It was also concluded that the uniform definition of passenger car equivalent is not applicable and that the values of equivalency depend on traffic composition, degree of saturation and location. Chandra and Sikdar [9] proposed a methodology to estimate PCE values for mixed traffic conditions. They estimated the PCE values as a function of vehicle area and speed. The study done by Ahmeda Al-Kaisy et al. [10] led to the conclusion that the effect of heavy vehicles on traffic is a lot more visible in congestion periods than in less saturated conditions. The effect of heavy vehicles on traffic is influenced by limiting factors. Al-Kaisy et al. [11] used queue discharge factor as a measure of performance to estimate PCE values during congestion. Bham and Benekohal [12] used percent space occupancy in their work to represent congested traffic conditions in a better way when traffic is composed of vehicles with different lengths. According to that, space occupancy provides a better feel for the traffic conditions since it incorporates the length of each vehicle and it is therefore a better parameter than the average length used in density. Chitturi and Benekohal [13] estimated the influence of work zones on PCE and concluded that the equivalent values are lower when the percent of heavy trucks is increased, as well as when traffic capacity is increased in zones. When it comes to motorcycles, Rongviriyapanich and Suppattrakul [14] derived $\mathrm{PCE}$ values for road sections near intersections and mid-block sections using time headways. They found that PCE of motorcycle continuously decreases with the increase in the proportion. Also, Ahmed [15] studied identification of heavy vehicle characteristics that have influence on the vehicle flow in different congestion conditions with emphasis on the level of service. In this study he derived PCE factors in congestion conditions and compared them with HCM-2000 models [3].

\section{METHODOLOGY}

The objective of our research as well as the basic steps used in the methodology are given and described in this section. The methodology steps include: determining PCE at cross sections, selection of measurement locations and it ends with collection and data synthesis.

\subsection{Objective of the research}

Motivation behind this research presents a hypothesis that the negative effect of heavy vehicles on the traffic conditions on two-lane roads is bigger with the increase of longitudinal gradient value (LG). This claim relates to all vehicles which do not belong to the passenger vehicles category.

The previous claim is based on the fact that the time headways are significantly increased with the increase of longitudinal gradient for vehicles which are not in the category of passenger vehicles. This fact needs to be taken into account in the capacity 
analysis, with regard to variable values of AADT (Annual Average Daily Traffic) [16]. Relevant literature related to analysis of heavy vehicle equivalent values mentions rather contradictory attitudes and due to that fact PCE values were radically changed from extremely high to extremely low [3, 17].

\subsection{Determining PCE at cross sections}

Seguin and associates [18] formulated a special method for calculating PCE, based on Greenshields model. This method defines PCE as a relation of mean value of time headways of the observed vehicle type divided with the mean value of headways made by passenger vehicles and is formulated as:

$E_{T}=\frac{H_{i j}}{H_{B}}$

where:

$H_{i j}$ - mean value of headways for the observed vehicle type, $i$ under conditions $j$ and

$H_{B}$-mean value of headways of passenger vehicles.

The same method can be applied not only for spatial but also for time headways (th).

Current engineering manuals use the so called "commercial vehicles impact factor" in the capacity analysis for the quantification of influence of the traffic flow structure on the saturated flow rate in the area of former Yugoslavia [19, 20]. This factor is essentially formed based on the PCE values of the defined categories of vehicles. Their relationship can be seen in the following relation:

$$
\begin{aligned}
F_{K V}= & 1 /\left[1+P_{i} \cdot\left(P A E_{i}-1\right)+P_{j} \cdot(P A E j-1)+\ldots\right. \\
& \left.+P_{n} \cdot\left(P A E_{n}-1\right)\right]
\end{aligned}
$$

where:

$F_{K V} \quad$ - commercial vehicle impact factor;

$P_{(i-n)} \quad$ - percentage of individual categories of vehicles in traffic flow;

$P A E_{(i-n)}$ - PCE value for a specific category of vehicles.

\subsection{Selection of measurement locations}

In order to ensure that the time headways (and PCE as well) depend only on the size of longitudinal gradient, the following criteria must be met:

- Geometric elements of situational plan and cross-section profile of two-lane road are such that they do not pose a limiting factor and that they enable movement of vehicles in a free traffic flow;

- There are no works on the road in the vicinity, overtaking is prohibited (full white centre line) and there are no settlements around;

- There are no cross-roads, side roads or other facilities by the road nearby before or after the measurement locations, which could interfere with the continuity of headway in the traffic flow;
- Cross-section profile contains minimum two lanes at least $3.0 \mathrm{~m}$ wide, with curbs and shoulders;

- Distance between two measurement locations on the ends of the selected road section with or without longitudinal gradient is not shorter than 1,000 metres.

- Continuity in size of longitudinal gradient is present before and after the measurement location with allowed deviation up to $\pm 0.5 \%$, long enough for the measuring time headways, i.e. PCE values.

Measurements were performed on two-lane state roads in Bosnia and Herzegovina at the locations shown in Figure 1. Basically, the length of the observed road sections was not the same for all routes, because some parts of the sections were selected from the existing road sections. The longitudinal gradient on individual road section parts was determined by calculating the longitudinal gradient Arithmetic Mean (AM) values for the given road section, which were measured at every $200 \mathrm{~m}$. In this way the observed parts of road sections were selected based on the mean value of the longitudinal gradient, and the measurement cross-section was located at the end of the observed part of the road section. The plan of the conducted research predicted the measurement of PCE values based on the time headways. As the basic PCE value in this research headway between passenger vehicles PV-PV, value 1 was taken, although PCE was not taken into consideration for different categories of passenger vehicles. Representative time headways for passenger vehicles are their arithmetic mean values

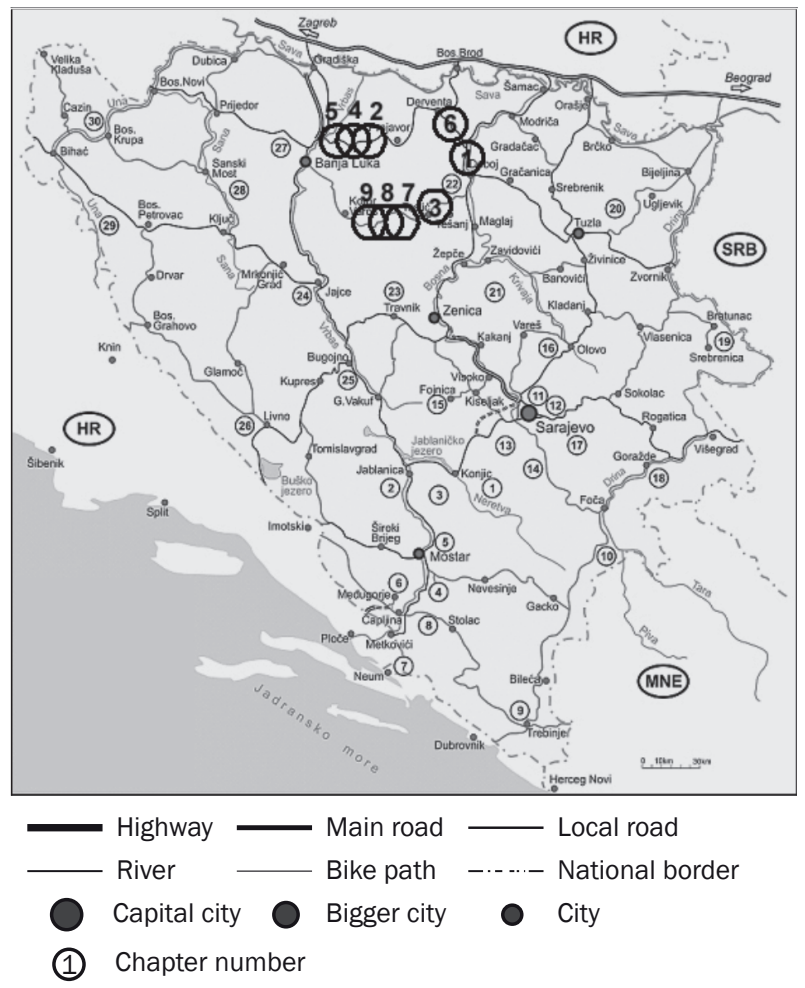

Figure 1 - Measurement locations 
measured at cross-sections, as shown in Table 1. The number of measured time headways at cross-section is given as $n-1$, because the first vehicle is not taken into consideration. Slight increase of AADT values on the observed parts of the selected road sections in the previous period has negligible influence on the measured time headway.

A fact that should be considered as well is that there were no additional traffic lanes for slow vehicles present on the observed measurement locations on the two-lane state roads which would disturb the measurement of PCE values.

\subsection{Collection and data synthesis}

Measurements were performed by software "PROTOK VOZILA“ v. 1.0.0.0 (Vehicle Flow, transl. note), made at the Faculty of Transport and Traffic Engineering in Belgrade, installed on a notebook computer, Intel Pentium Dual CPU $2.16 \mathrm{GHz} 2 \mathrm{~GB}$. The measured time headways were recorded in the database, according to vehicle category determined based on the operator's free estimate. Relevant data were recorded in a notepad document which was transferred into Microsoft Office Excel and processed after measurements were completed.

The software "PROTOK VOZILA", during recording of time headways values onto a computer immediately performs the classification of measured data into five categories: Passenger Vehicles (PV), Light Vehicles (LV), Heavy-Duty Vehicles (HDV), Buses (BUS) and Auto Trains (AT), following five categories given at the beginning of this section.

When talking about the number of measured time headways (and therefore PCE values) of certain vehicle categories at a measurement location, the aim is to collect minimal statistical sample of more than 100 commercial vehicles, which was achieved in most of the cases. The recorded number of vehicles at characteristic measurement locations grouped by category is shown in Table 2.

For each measurement location, PCE values were calculated for every vehicle in each observed category, based on the recorded time headways. After that, the values of relevant statistical parameters which include the arithmetic mean, standard deviation (SD) and variation coefficient (VC) were determined. Vehicles in each category (LV, HDV, BUS, AT and all PV) were then classified according to PCE values into statistical classes 0.5 units in width in order to get empirical distribution of passenger vehicle equivalents. Software TableCurve 2D v5.01 was used for regression analysis. After calculating AM, SD and VC we have determined the PCE models for all categories of vehicles depending on the gradient. Figure 2 presents standard deviation numbers depending on the longitudinal gradient. The models were tested on the second-degree polynomial function expressed as:

$y=A \cdot x^{2}+B \cdot x+C$

where:

$y=P C E$,

$X \quad$-gradient

$A, B, C$-parameters obtained by regression analysis.

The performed statistical tests have shown acceptable values of correlation coefficient, especially for PCE values on higher road gradients. By testing the model of a second-degree polynomial function, higher values of correlation coefficient were determined, but dependency curves have shown an unacceptable form. The data grouped in statistical classes were used for further analysis which included determining validity of distribution and determining percentile value of passenger car equivalents $\left(\mathrm{PCE}_{15 \%}, \mathrm{PCE}_{50 \%}\right.$ and $\left.\mathrm{PCE}_{85 \%}\right)$.

Cumulative distribution was used for determining $\mathrm{PCE}_{15 \%}, \mathrm{PCE}_{50 \%}$ and $\mathrm{PCE}_{85 \%}$, in order to find function square root for increment 0.01 . From the resulting
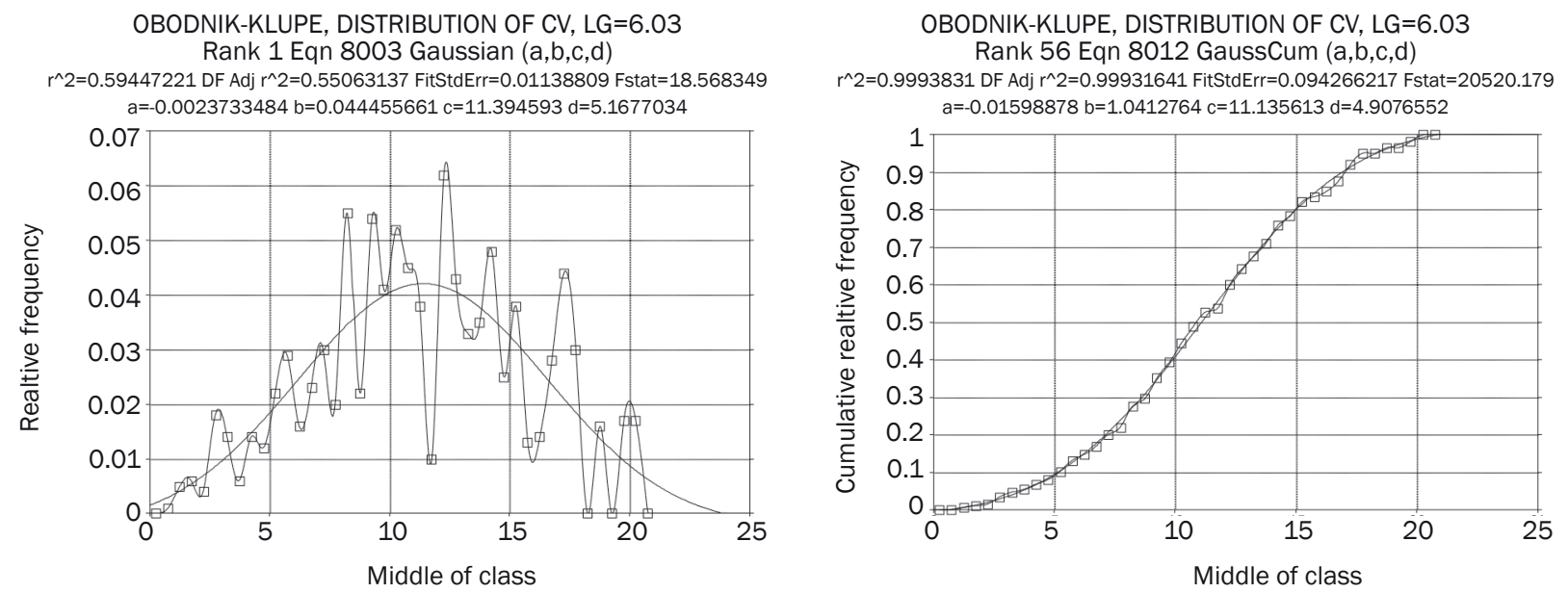

Figure 2 - Empirical distribution analysis of PCE values 


\begin{tabular}{|c|c|c|c|c|c|c|c|c|c|}
\hline$\underset{\stackrel{\Phi}{F}}{F}$ & $\begin{array}{l}\stackrel{\leftrightarrow}{0} \\
\ddot{m}\end{array}$ & $\begin{array}{l}\stackrel{\Omega}{2} \\
\stackrel{\sigma}{2}\end{array}$ & $\begin{array}{l}\underset{N}{ } \\
\stackrel{\sim}{N}\end{array}$ & $\begin{array}{l}\text { @) } \\
\text { ָे }\end{array}$ & $\begin{array}{l}\infty \\
\stackrel{1}{1} \\
\infty\end{array}$ & $\begin{array}{l}\stackrel{R}{R} \\
\dot{f}\end{array}$ & $\begin{array}{l}\text { ণ़ } \\
\text { ते }\end{array}$ & $\begin{array}{l}\underset{T}{A} \\
\dot{A}\end{array}$ & $\begin{array}{l}0 \\
0 \\
0 \\
0\end{array}$ \\
\hline 웜 & 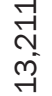 & $\begin{array}{l}\mathscr{8} \\
\stackrel{7}{-1} \\
i\end{array}$ & 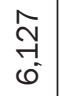 & $\begin{array}{l}0 \\
\stackrel{2}{7} \\
\text { if }\end{array}$ & $\begin{array}{l}0 \\
\text { न } \\
6 \\
6\end{array}$ & $\begin{array}{l}0 \\
0 \\
6 \\
\dot{f}\end{array}$ & $\begin{array}{l}\infty \\
\infty \\
\infty \\
\infty \\
\end{array}$ & $\begin{array}{l}\infty \\
\stackrel{\infty}{\infty} \\
\infty \\
\infty \\
\end{array}$ & $\begin{array}{l}\infty \\
\infty \\
\infty \\
\infty \\
n^{0}\end{array}$ \\
\hline $\begin{array}{l}\text { ஜे } \\
\text { ஜे }\end{array}$ & 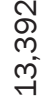 & 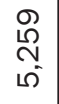 & 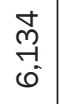 & 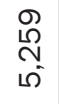 & $\begin{array}{l}\stackrel{\Omega}{\Omega} \\
\stackrel{N}{ } \\
\text { م) }\end{array}$ & $\begin{array}{l}\vec{m} \\
o \\
\stackrel{\forall}{*}\end{array}$ & $\begin{array}{l}\mathbb{N} \\
\infty \\
\text { ñ }\end{array}$ & $\begin{array}{l}\mathbb{N} \\
\infty \\
\infty\end{array}$ & $\begin{array}{l}\widetilde{W} \\
\infty \\
\end{array}$ \\
\hline$\stackrel{\infty}{\stackrel{\sim}{~}}$ & 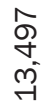 & $\begin{array}{l}8 \\
8 \\
\circ \\
10\end{array}$ & $\begin{array}{l}\infty \\
\infty \\
\infty \\
10\end{array}$ & $\begin{array}{l}8 \\
8 \\
\circ \\
10\end{array}$ & $\begin{array}{l}8 \\
\circ \\
\text { ம) }\end{array}$ & $\begin{array}{c}\stackrel{2}{N} \\
\infty \\
+\end{array}$ & $\begin{array}{l}\Omega \\
\hat{0} \\
\dot{m}\end{array}$ & $\begin{array}{l}\Omega \\
\hat{0} \\
\dot{m}\end{array}$ & $\begin{array}{l}\Omega \\
\hat{0} \\
\text { n' }\end{array}$ \\
\hline 豆 & 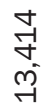 & 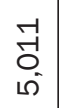 & $\begin{array}{l}\llcorner 0 \\
\infty \\
\infty \\
10\end{array}$ & $\begin{array}{l}-1 \\
0 \\
0 \\
10\end{array}$ & \begin{tabular}{l}
-1 \\
\multirow{-}{0}{} \\
10
\end{tabular} & $\begin{array}{l}N \\
\infty \\
\sim \\
\forall\end{array}$ & $\begin{array}{l}\stackrel{8}{0} \\
\dot{0} \\
\dot{m}\end{array}$ & $\begin{array}{l}\text { : } \\
\dot{0} \\
\dot{m}\end{array}$ & $\begin{array}{l}8 \\
8 \\
\dot{0}\end{array}$ \\
\hline $\begin{array}{l}\stackrel{0}{\circ} \\
\stackrel{\sim}{ }\end{array}$ & 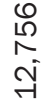 & $\begin{array}{l}\stackrel{L}{\llcorner} \\
\stackrel{\infty}{\infty} \\
\stackrel{+}{*}\end{array}$ & 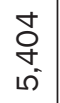 & $\begin{array}{l}\llcorner \\
\stackrel{\infty}{\infty} \\
\infty \\
\forall\end{array}$ & 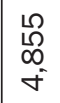 & 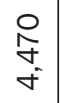 & $\begin{array}{l}\stackrel{m}{f} \\
\stackrel{m}{m}\end{array}$ & \begin{tabular}{l}
$\stackrel{m}{f}$ \\
\multirow{\leftarrow}{m}{} \\
$\dot{m}$
\end{tabular} & $\begin{array}{l}\stackrel{m}{+} \\
\stackrel{n}{m}\end{array}$ \\
\hline ஜ & 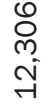 & $\begin{array}{l}\hat{D} \\
\infty \\
+ \\
+\end{array}$ & 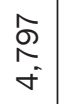 & $\begin{array}{l}\text { D. } \\
\infty \\
+\end{array}$ & $\begin{array}{l}\hat{S} \\
\infty \\
+ \\
+\end{array}$ & 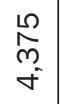 & $\begin{array}{l}\stackrel{\llcorner}{N} \\
\text { n் }\end{array}$ & 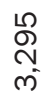 & $\begin{array}{l}\stackrel{L}{\Omega} \\
\stackrel{N}{n} \\
\text { n. }\end{array}$ \\
\hline 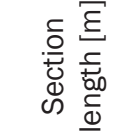 & $\begin{array}{l}8 \\
\infty \\
\infty \\
i\end{array}$ & $\begin{array}{l}8 \\
0 \\
\stackrel{-}{-}\end{array}$ & $\begin{array}{l}8 \\
: \\
i\end{array}$ & $\begin{array}{l}8 \\
\infty \\
\rightarrow \\
-1\end{array}$ & $\begin{array}{l}8 \\
: \\
i\end{array}$ & $\begin{array}{l}8 \\
\stackrel{-}{-} \\
-\end{array}$ & 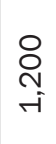 & 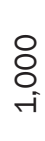 & 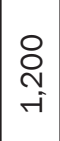 \\
\hline 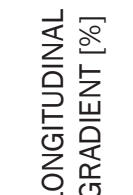 & ¿ & $\underset{\text { \& }}{\stackrel{8}{ }}$ & $\begin{array}{l}\hat{O} \\
\stackrel{i}{v}\end{array}$ & $\begin{array}{l}\stackrel{\text { }}{\text { mे }} \\
\text {. }\end{array}$ & $\stackrel{\leftrightarrow}{\stackrel{一}{ }}$ & $\begin{array}{l}8 \\
i \\
i \infty\end{array}$ & $\begin{array}{l}m \\
0 \\
0\end{array}$ & 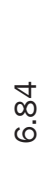 & $\stackrel{\text { L }}{\stackrel{\text { f }}{*}}$ \\
\hline 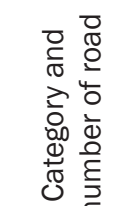 & 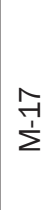 & $\begin{array}{l}-1 \\
\underline{\theta} \\
\stackrel{1}{\Sigma}\end{array}$ & $\stackrel{\nabla}{\Sigma}^{+}$ & $\begin{array}{l}-1 \\
\stackrel{9}{0} \\
\stackrel{1}{2}\end{array}$ & $\begin{array}{l}-1 \\
\stackrel{9}{6} \\
\stackrel{-1}{\Sigma}\end{array}$ & \begin{tabular}{l} 
N \\
\multirow{7}{*}{} \\
$\sum$
\end{tabular} & $\sum_{\Sigma}^{+t}$ & $\sum_{i}^{+}$ & $\sum_{\Sigma}^{\nabla}$ \\
\hline 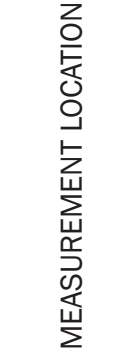 & 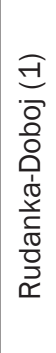 & 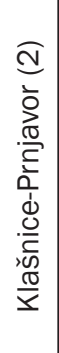 & 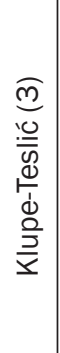 & 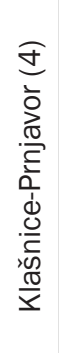 & 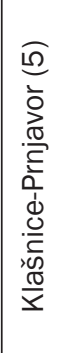 & 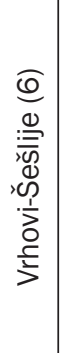 & 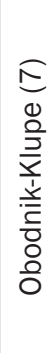 & 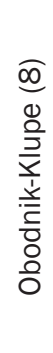 & 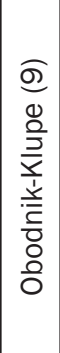 \\
\hline
\end{tabular}

\begin{tabular}{|c|c|c|c|c|c|c|c|c|c|c|}
\hline 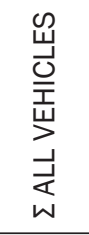 & 总 & नै & 产 & ه্ & ¿े & ○̊ & $\begin{array}{l}\dot{p} \\
\stackrel{n}{\sim}\end{array}$ & $\frac{\mathfrak{s}}{\mathrm{i}}$ & & ty \\
\hline $\begin{array}{l}\frac{\partial}{d} \\
\frac{1}{\alpha}\end{array}$ & 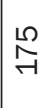 & 극 & $\stackrel{\infty}{\underset{\sim}{\sim}}$ & $\vec{m}$ & 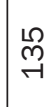 & $\stackrel{\infty}{\stackrel{\infty}{\sim}}$ & 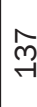 & శి & & 弟 \\
\hline 安 & สิ & 0 & $\sigma$ & $r$ & $r$ & $\stackrel{m}{\sim}$ & $\stackrel{\varphi}{r}$ & 17 & & ? \\
\hline$\stackrel{\mathscr{N}}{\mathrm{D}}$ & $\stackrel{\rightarrow}{\rightarrow}$ & $\stackrel{\leftrightarrow}{\neg}$ & $\stackrel{d}{\text { t }}$ & ᄀ & ᄀ & 7 & 7 & 7 & & - \\
\hline 로 & $\mathscr{C}$ & $\stackrel{\text { F }}{*}$ & ले & 吕 & م & $\stackrel{\infty}{m}$ & $\stackrel{\infty}{m}$ & 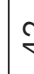 & & Rि \\
\hline$\geq$ & $\stackrel{\infty}{\sim}$ & F & 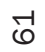 & เి & กิ & $\infty$ & 8 & $\mid \begin{array}{l}0 \\
b\end{array}$ & & $\hat{0}$ \\
\hline a & $\underset{\infty}{\stackrel{్}{~}}$ & $\begin{array}{l}\text { No } \\
\infty\end{array}$ & ల్లి & $\stackrel{\hat{N}}{\hat{N}}$ & $\begin{array}{l}\infty \\
0 \\
\infty\end{array}$ & 点 & 条 & $\bar{\delta}$ & & 竞 \\
\hline 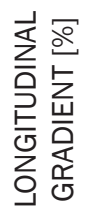 & $\begin{array}{l}8 \\
\vdots\end{array}$ & $\begin{array}{l}8 \\
\text { ¿ }\end{array}$ & 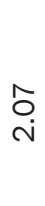 & i़ & $\underset{+}{\stackrel{8}{ }}$ & $\begin{array}{l}8 \\
\text { ¿ }\end{array}$ & $\begin{array}{l}m \\
0 \\
0\end{array}$ & g & & fُ \\
\hline 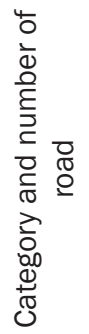 & 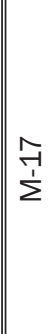 & 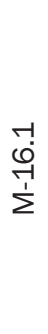 & $\sum_{\Sigma}^{+}$ & 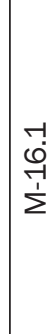 & $\mid \begin{array}{l}-1 \\
0 \\
\dot{0} \\
\dot{2}\end{array}$ & $\frac{7}{7}$ & $\stackrel{+}{\Sigma}$ & $\sum$ & & $\stackrel{+}{\Delta}$ \\
\hline 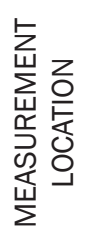 & 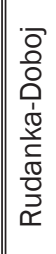 & 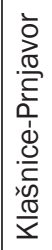 & 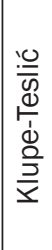 & 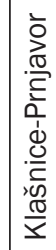 & 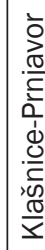 & 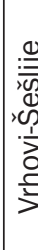 & 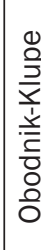 & 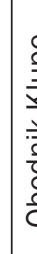 & & 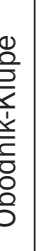 \\
\hline
\end{tabular}


values of function root for increment 0.01 , values of function root $x$ for values $y(0.15,0.50$ and 0.85$)$ were separated, which basically represent those percent values of PCE. The performed statistical tests have included analysis on normal (Gaussian) and log-normal distributions. Although it is assumed that the PCE empirical distribution, in cases of sufficiently large statistical sample, can be approximated with theoretical normal distribution, that is not of importance for the scope of this research. The most important step in the performed statistical analysis was the determination of cumulative distribution function, which, as it turned out, in all cases can be approximated with normal cumulative (Gaussian) distribution.

\section{ANALYSIS AND DISCUSSION OF THE RESULTS}

From the diagram shown in Figure 3 it is obvious that the maximum measured road gradient value for two-lane roads is $7.45 \% \cong 7.50 \%$ and that PCE value for AT vehicle category on road gradient of $7.5 \%$, exceeds the value of 16 . In the case where road gradient is equal to $0(0 \%)$, the only PCE value with significant deviation is determined in AT vehicle category, while other values are approximately equal. Therefore, PCE value for AT vehicle category, grows significantly more progressively with the increase of longitudinal road gradient compared to PCE values of other vehicle categories. On longitudinal road gradient of $7.5 \%$, deviation of measured PCE values in AT and LT vehicle categories is more than 10 units.

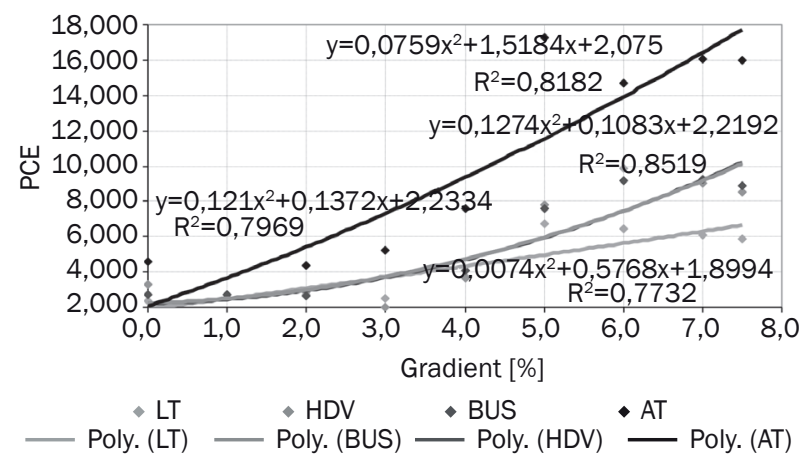

Figure 3 - Model for determining PCE values depending on the road gradient for different categories of vehicles in Bosnia and Herzegovina

The obtained correlation coefficient values show satisfactory strength level of correlation between PCE values and longitudinal gradient. Based on the analysis of PCE values depending on the road gradient, the maximum value of correlation coefficient is determined for BUS vehicle category, whereas the minimal correlation coefficient value is determined for all types of commercial vehicles (LT, HDV, BUS and AT). This can be explained by the fact that PCE values on the longitudinal gradient are largely dependent on the drivers' behaviour, which implies that the bus drivers have a relatively high level of professionalism in relation to other driver categories. This also explains bigger deviation of time headways (i.e. PCE values) within LT vehicles category on the longitudinal gradient, and minimum deviation of PCE values within BUS vehicle category. On the upward road gradient this loss is frequently explained with the vehicle structure, (for heavy vehicles load weight has also additional impact). Lower correlation coefficient values on two-lane state roads are the results of a large deviation of PCE values, which is noticed for all commercial vehicles. Also, higher deviations of PCE values were determined on the measurement location Obodsnik-Klupe, which, unlike other observed locations, is within mountainous terrain.

PCE comparative values for all commercial vehicle categories, on $\mathrm{PCE}_{15 \%}, \mathrm{PCE}_{50 \%}$ and $\mathrm{PCE}_{85 \%}$ models are presented in the following diagrams (Figures 4, 5 and 6).

The obtained PCE values for $\mathrm{PCE}_{15 \%}$ model (Figure 4), show a significant deviation of PCE value for AT vehicle category with the increase of longitudinal road gradient value. It is also noticed that by the increase of longitudinal grade value, PCE gains higher values within BUS than within HDV vehicle category, which can be interpreted by more cautious driving of BUS drivers in the mountainous areas.

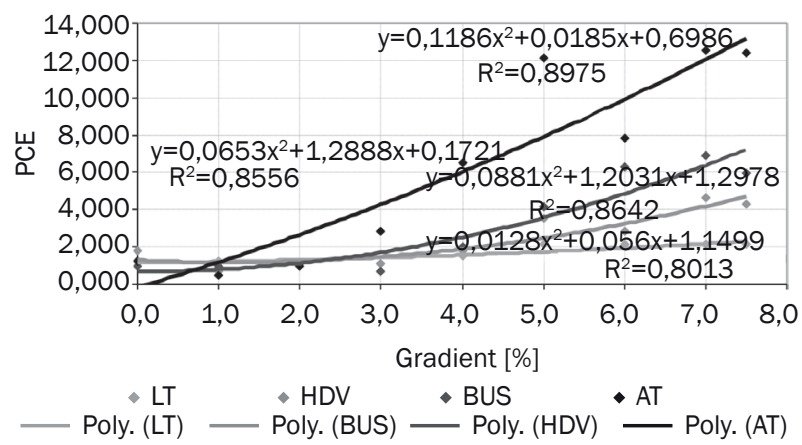

Figure 4 - $P C E_{15 \%}$ model depending on the longitudinal road gradient for different categories of vehicles

The obtained PCE values for $\mathrm{PCE}_{50 \%}$ model (Figure 5), show minimal deviation of PCE value on road gradient values between 0 and 2 percent. In cases where longitudinal road gradient values are equal or higher than $2 \%$, the deviation of PCE values in the observed vehicle categories is also increased. It is also determined that in cases of road gradient value of $5 \%$, PCE values in BUS and LV vehicle category become almost equal.

In the case where longitudinal road gradient value is equal to $1 \%$, PCE value becomes approximately equal in AT and BUS vehicle categories. Similarly, approximately equal values are determined within LV 


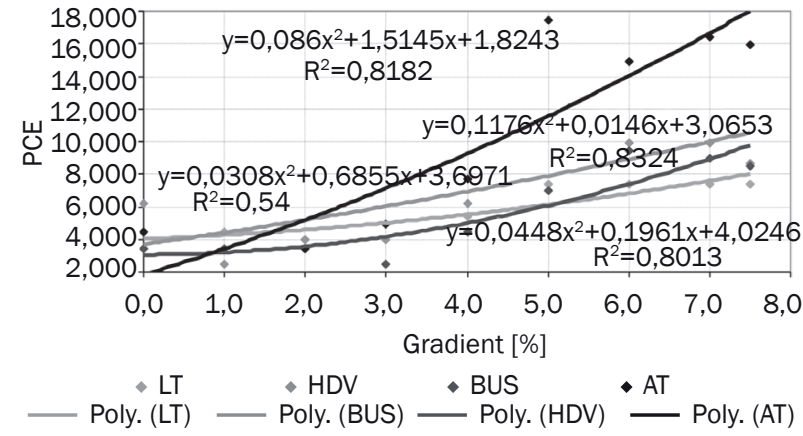

Figure 5 - $P C E_{50 \%}$ model depending on the longitudinal road gradient for different categories of vehicles

and HDV vehicle categories. It is also noticed that PCE value is approximately equal for LT and AT vehicle category in cases where longitudinal road gradient is equal to $2 \%$. More significant deviation of PCE value for LT, HDV, BUS and AT vehicle categories is determined for longitudinal road gradient values equal to or higher than 3\% (Figure 6). The resulting curve for all commercial vehicles is slightly above the curve for AT vehicle category in cases of higher longitudinal road gradients.

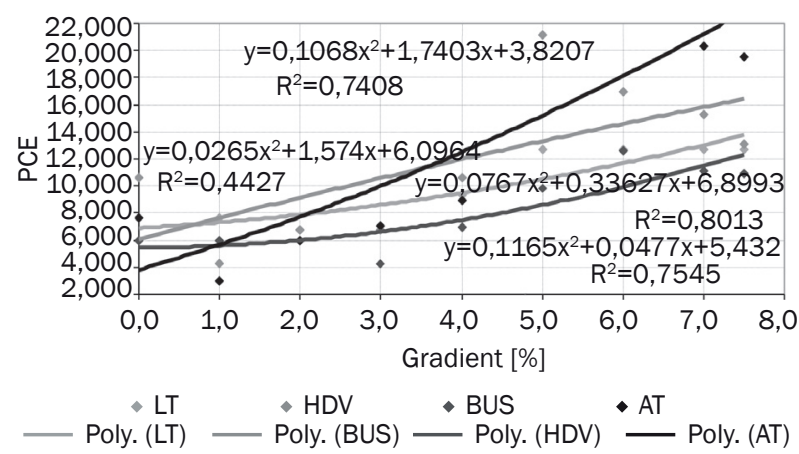

Figure 6 - $P C E_{85 \%}$ model depending on the longitudinal road gradient for different categories of vehicles

In order to compare the obtained results with the values given in HCM it was necessary to determine HCM model PCE values for the whole range of a longitudinal road gradient in the form of a curve (for each version of $\mathrm{HCM}$ ). This was achieved by creating comparative models based on the values recommended in $\mathrm{HCM}$, for the selected road sections and traffic conditions (mountainous terrain, vehicle flow, consolidating BUS+HDV vehicle categories etc.), which were then used for comparative analysis of $\mathrm{HCM}$ models PCE values with PCE values determined based on the data collected in this research. It was also necessary to adjust the obtained models considering the prevailing conditions in the field. There are also certain differences between individual HCM models. HCM-2000 model [3] examines the traffic flow (in order to form the models it is necessary to analyse AADT values for the given road section) while HCM-2010 model [17] consolidates trucks and BUSes into the category of trucks, etc. Considering all these parameters, the models for determining PCE values were formed, adjusted for the prevailing characteristics of measurement locations selected in this research. Although the values obtained by adjusting the models can frequently be the approximation of transferring a model into unreal conditions, in this case maximally authentic parameters are taken which describe that model. In this way, by selecting appropriate PCE values based on HCM models $[3,17]$, representative comparative values were determined which were then used for comparative analysis of HCM models with models from this research.

By comparing research results with the two latest versions of HCM models [3] and [17] (see Figure 7), it is noticed that PCE values determined in our models overlap with PCE values obtained from HCM-2000 model [3] for the longitudinal road gradient value of $2.5 \%$, while in case of HCM-2010 model [17], the resulting PCE values overlap for longitudinal road gradient value of $1.5 \%$. In cases of higher longitudinal road gradient values (for HCM-2000 LG 2.5\%, i.e. HCM2010 LG 1.5\%) larger PCE values are obtained from $\mathrm{HCM}$ models, than from models formulated in this research. Based on the research results it can be concluded that for longitudinal road grade values equal or higher than 3\%, PCE values obtained by performed measurements have lower values than PCE values determined in the observed HCM models $[3,17]$. Therefore, the difference of PCE values obtained from HCM2000 and HCM-2010 models for the given conditions on a longitudinal road gradient of $7.5 \%$ is from 2.5 to 3 units. It is obvious that PCE values for a given local traffic and road conditions observed in Bosnia and Herzegovina are not adjusted with PCE values in HCM models.

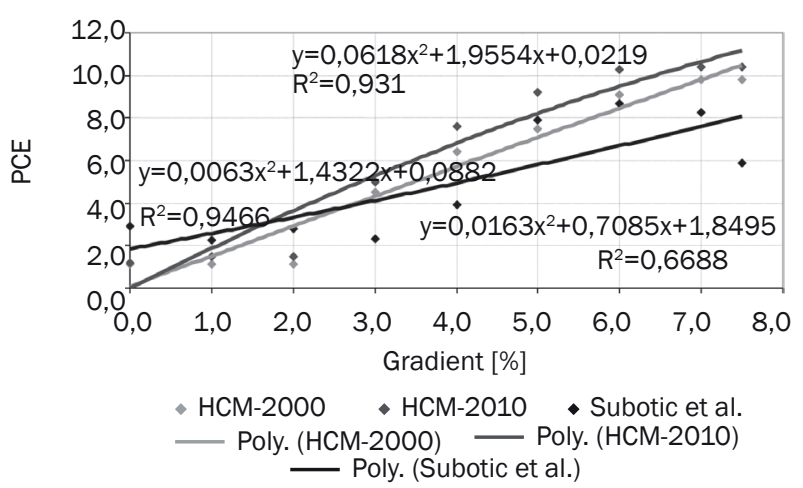

Figure 7 - Comparative analysis of PCE obtained values with HCM-2000 and 2010

\section{CONCLUSION}

The research results confirm the starting hypothesis that the effect of heavy vehicles on traffic conditions on two-lane roads increases in cases of higher longitudinal road gradient values. PCE values depend 
on advancements in the technology of making and producing new vehicles, as well as on driving and dynamic characteristics of vehicles. The data obtained from this research show lower PCE values for all vehicle categories compared to observed Highway Capacity Manual models.

The arithmetic mean of PCE values for LT, HDV, BUS, AT vehicle categories is grouped between values 2 and 3, except for the PCE value for AT vehicle category which is higher than 4. Deviation of PCE value for AT vehicle category from PCE values determined in other vehicle categories is higher with the increase of a longitudinal road gradient. Hence, it was determined that with the increase of longitudinal road gradient, PCE value is also increased for all vehicle categories, and depending on the category of vehicles PCE values decrease, which is especially shown in correlation coefficient R2=0.6688 for all vehicles (see Figure 7). On the Obodnik-Klupe road section with longitudinal road grade of $7.5 \%$, the obtained PCE values deviate by more than 10 units between AT and LT vehicle categories, which can be explained with the presence of mountainous terrain where larger PCE variations occur. Minimum PCE value for AT vehicle category with longitudinal road gradient of $1 \%$ is 2 , and for the longitudinal road gradient of $7.5 \%$, the resulting PCE value exceeds the value of 16 , which in itself shows significant variation of the PCE value depending on the percentage of longitudinal road gradient.

When PCE values from this paper were compared to other HCM models (2000 and 2010) it was concluded that PCE values significantly vary and that in cases of longitudinal road grade values equal or higher than $3 \%$ models formulated in this research give lower PCE values for all commercial vehicles than observed $\mathrm{HCM}$ models.

Based on the generalized model (see Figure 7), it is proved that by increasing longitudinal road gradient value, the resulting PCE gradient curve becomes almost linear but the correlation coefficient becomes lower, which indicates high fluctuations of PCE values on higher longitudinal road gradients. Maximum PCE value for commercial vehicles on a two-lane road, in observed traffic conditions on longitudinal road grade values equal or higher than $7 \%$ is 8 , which shows significant influence of commercial vehicles on the movement of passenger vehicles in traffic flow.

One of the limitations of this paper is that the PCE estimation, using time headway method should be estimated at normal and saturated traffic flow conditions, not just free flow conditions.

Research results indicate the need for constant monitoring of vehicle structure characteristics, and time headways of vehicles in the traffic flow. In order to collect relevant information regarding the vehicle structure and the performance of certain categories of vehicles, it is necessary to conduct similar studies in the future periods, since it can be expected that the structure of vehicles will be significantly changed. Also, the effect of variation in traffic flow on PCE could be considered in some further research.

\section{Dr MARKO SUBOTIĆ ${ }^{1}$}

E-mail: msubota@gmail.com

Dr VLADAN TUBIĆ ${ }^{2}$

E-mail: vladan@sf.bg.ac.rs

1 Univerzitet u Istočnom Sarajevu, Saobraćajni fakultet

Vojvode Mišića 52, 74000 Doboj, Bosna i Hercegovina

2 Univerzitet u Beogradu, Saobraćajni fakultet Vojvode Stepe 305, 11000 Beograd, Srbija

\section{ZAVISNOST EKVIVALENATA VOZILA OD UZDUŽNOG NAGIBA NA DVOTRAČNIM PUTEVIMA BOSNE I HERCEGOVINE}

\section{ABSTRAKT}

Glavni cilj ovog rada je da se na osnovu obimnih empirijskih istraživanja u slobodnom toku u lokalnim uslovima kvantificira nepovoljni uticaj strukture toka kroz vrednosti ekvivalenata (PCE) u funkciji uzdužnog nagiba. Na osnovu dostupnih literarnih izvora i empirijskih istraživanja dokazano je da je PCE vrednost u direktnoj vezi sa uzdužnim nagibom za bilo koju klasu vozila. Utvrđene su vrednosti PCE u slobodnom toku za usvojene klase vozila. Na osnovu izmerenih vrednosti razvijeni su modeli za prosečnu vrednost PCE u funkciji uspona za dvotračni put. Poređenjem dobijenih modela u slobodnom toku sa HCM priručnicima pokazano je da je u ovom istraživanju PCE vrednost manja. Takođe su uspostavljeni i modeli procentnih PCE vrednosti $P C E_{15 \%}$, $P C E_{50 \%}$ i $P C E_{85 \%}$

\section{KLUUČNE REČI}

ekvivalent putničkih vozila; nivo usluge; analiza kapaciteta dvotračnog puta; regresiona analiza;

\section{REFERENCES}

[1] Rahka H, Lucic I. Variable Power Vehicle Dynamics Model for Estimating Truck Accelerations. Journal of Transportation Engineering. 2002;128(5):412-419.

[2] Kockelman K. Changes in the Flow-Density Relation due to Environmental, Vehicle and Driver Characteristics. Transportation Research Record. 1998;1644:47-56.

[3] Highway Capacity Manual. Transportation Research Board. Washington D.C.: National Research Council; 2000.

[4] Hranac R, Sterzin E, Krechmer D, Rakha H, Farzaneh M. Empirical Studies on Traffic Flow in Inclement Weather. U.S. Department of Transportation: Federal Highway Administration, 2006; Publication No. FHWAHOP-07-073.

[5] Al-Kaisy A, Jung Y, Rakha H. Developing Passenger Car Equivalency Factors for Heavy Vehicles during Congestion. Journal of Transportation Engineering. 2005;131(7):514.

[6] Elefteriadou L, Torbic D, Webster N. Development of passenger car equivalents for freeways, two-lane highways and arterials. Transportation Research 
Record: Journal of Transportation Research Board. 1997;1572:51.

[7] Bhuvanesh S. Simulation and Animation of Heterogeneous Traffic on Urban Roads [Ph. D. Thesis]. Indian Institute of Technology, Kanpur, India; 1999.

[8] Khan SI, Maini P. Modeling Heterogeneous Traffic Flow. Transportation Research Record: Journal of Transportation Research Board. 1999;1678:234.

[9] Chandra S, Sikdar PK. Factors affecting PCU in mixed traffic on urban roads. Road Transport Research. 2000;9(3):40.

[10] Al-Kaisy A, Hall F, Reisman E. Developing passenger car equivalents for heavy vehicles on congested freeways: A capacity based approach. Presented at the 80th Annual Meeting of the Transportation Research Board. Transportation Research Board, Washington, D.C.; 2001.

[11] Al-Kaisy A, Hall F, Reisman E. Developing Passenger Car Equivalents for Heavy Vehicles on freeways during queue discharge flow. Transportation Research: Part A. 2002;36(8):725-742.

[12] Bham GH, Benekohal RF. A High Fidelity Traffic Simulation Model based on Cellular Automata and Car-Following concepts. Transportation Research Part C: Emerging Technologies. 2004;12(1):1-32.

[13] Chitturi MV, Benekohal RF. Passenger car equivalents for heavy vehicles in Work Zones. Presented at the $87^{\text {th }}$ Annual Meeting of the Transportation Research
Board. Transportation Research Board, Washington, D.C.; 2007.

[14] Rongviriyapanich T, Suppattrakul C. Effects of motorcycles on traffic operations on arterial streets. Journal of Eastern Asia Society for Transportation Studies. 2005;6:137-146.

[15] Ahmed U. Passenger Car Equivalent Factors for Level Freeway Segments Operating under Moderate and Congested Conditions [Master's Thesis]. Marquette University. Milwaukee, Wisconsin; 2009.

[16] Štefančić G, Marijan D, Kljajić S. Capacity and Level of Service on the Zagreb bypass. Promet - Traffic \& Transportation. 2012;24(3):261-267.

[17] Highway Capacity Manual. Transport Research Board Publications. Volume 4. Applications Guide; 2010.

[18] Seguin E, Crowley K, Zweig W. Passenger Car Equivalents on Urban Freeways. Report DTFH61-80-C-00106, FHWA. U.S. Department of Transportation; 1982.

[19] Čelar N, Istraživanje vrednosti zasićenog saobraćajnog toka na osnovnoj uličnoj mreži grada Beograda, Tehnika - Saobraćaj, 2007;54(6):1-6.

[20] Subotić M, Tubić V, Anđelković D. Research of the light duty vehicles equivalents at the mixed lanes of the signal crossroads at the central city zone. Mechanics Transport Communications: $20^{\text {th }}$ International Scientific Conference. 2011;(3):V1-V7. 